\section{Tumores cardíacos primarios}

\author{
ROBERTO GONZÁLEZ L. ${ }^{1,2}$, CLAUDIO TOLOZA A. ${ }^{\mathrm{a}}$, \\ RODRIGO REYES M. ${ }^{1,2}$, LORETO SPENCER L. ${ }^{3}$, \\ ENRIQUE SEGUEL S. ${ }^{2,3}$, ALECK STOCKINS L. ${ }^{1,2}$, \\ ANDRÉS JADUE T. ${ }^{1}$, ALEJANDRA RIQUELME U. ${ }^{4}$, \\ MATÍAS ÁVALOS T. ${ }^{a}$, SEBASTIÁN BARRA M. ${ }^{a}$, \\ FELIPE ALARCÓN O. ${ }^{a}$, EMILIO ALARCÓN C. ${ }^{1,2}$
}

\section{Primary cardiac tumors. Experience in 72 cases}

Background: Primary cardiac tumors have a low incidence, and their presentation form and clinical course are not well known. Aim: To describe the characteristics, treatment, follow up and survival of patients with primary cardiac tumors (PCT). Material and Methods: Review of all surgical procedures for PCT performed between 1984 and 2019 in a regional general hospital. The information was obtained from surgical protocols, surgical and pathology databases, medical records and registries of the Chilean National Identification Service. The clinical features of patients, immediate and long term outcomes are described. Results: Seventy-two surgical procedures for PCT were reviewed. Patients' age was $55 \pm 15$ years and $60 \%$ were women. The most common histological types were myxoma in 49 patients (68\%), papillary fibroelastoma in 13 (18\%) and sarcoma in 6 (8.3\%). Forty-nine (68.1\%) were symptomatic and all tumors were found on echocardiography. Fifty-one (71\%) were in the left atrium, 10 (14\%) in the aortic valve and eight (11\%) in the right atrium. The surgical procedures were tumor resection in 48 patients $(67 \%)$ and resection and repair with patch in 23 (32\%). Mean postoperative stay was $6.6 \pm 4.4$ days, eight patients (11\%) had complications and no patient died in the immediate postoperative period. Long term survival was higher in patients with benign PCT as compared with those with malignant tumors. Conclusions: Most PCTs in this group of patients were benign neoplasms and the most common tumors were myxomas. The main diagnostic method is echocardiography and the prognosis of surgical treatment is excellent when the tumors are benign.

(Rev Med Chile 2020; 148: 327-335)

Key words: Cardiac Surgical Procedures; Heart Neoplasms; Myxoma; Sarcoma.
${ }^{1}$ Centro Cardiovascular, Hospital Clínico Regional de Concepción "Dr. Guillermo Grant Benavente". Concepción, Chile.

Departamento de Cirugía, Facultad de Medicina, Universidad de Concepción. Concepción, Chile.

3Unidad de Anatomía Patológica, Hospital Clínico Regional de Concepción "Dr. Guillermo Grant Benavente". Concepción, Chile.

${ }^{4}$ Servicio de Cirugía, Hospital Clínico Regional de Concepción "Dr. Guillermo Grant Benavente". Concepción, Chile.

aEstudiante de Medicina, Facultad de Medicina, Universidad de Concepción. Concepción, Chile.

Trabajo no recibió financiamiento. Los autores declaran no tener conflictos de interés.

Recibido el 4 enero de 2019 , aceptado el 18 marzo de 2020.

Correspondencia a: Roberto González Lagos Departamento de Cirugía, Facultad de Medicina, Universidad de Concepción, Janequeo esquina Chacabuco S/N. Concepción, Chile. rgonzalezlagos@udec.cl
$\mathrm{D}$ esde que se conoce su existencia, los tumores cardiacos se han caracterizado tanto por su baja frecuencia, como por constituir un desafío diagnóstico y terapéutico ${ }^{1}$. Diferentes estudios de autopsias han reportado una prevalencia no mayor a $0,02 \%$ en la población general, destacando que solo $5 \%$ del total de las neoplasias que afectan al corazón corresponden a tumores cardiacos primarios (TCP $)^{2-4}$.
Aproximadamente $75-80 \%$ de los TCP son benignos y más de la mitad de ellos corresponde a mixomas, describiéndose también tipos histológicos menos frecuentes como: fibroelastoma papilar (FP), lipoma, fibroma, hemangioma, entre otros ${ }^{5-7}$.

La presentación clínica más frecuente es la insuficiencia cardiaca, siendo el síntoma más común la disnea, aunque también pueden presentarse 
embolias, alteración de la conducción cardiaca, síndromes constitucionales e incluso, muerte súbita $^{8}$. Los TCP también pueden ser asintomáticos, en cuyo caso habitualmente son pesquisados como un hallazgo ecocardiográfico incidental, durante el intraoperatorio de cirugía cardiaca o en el estudio post mortem 9 .

El estudio diagnóstico de elección en los TCP es la ecocardiografía, ya que, proporciona información acerca del tamaño, extensión, ubicación y repercusión funcional y hemodinámica. No obstante, existen ocasiones en que pueden ser requeridos estudios complementarios con resonancia magnética, tomografía computada e incluso tomografía por emisión de positrones para lograr una mejor identificación del tumor y de su extensión extra cardiaca ${ }^{10}$.

El tratamiento de los TCP es quirúrgico y dependiendo del tipo histológico, tamaño y contexto clínico puede ser desde resecciones simples, que incluyen solo la exéresis del tumor primario, hasta la necesidad de técnicas más complejas como el trasplante cardiaco ${ }^{11}$.

Con el desarrollo y disponibilidad de la ecocardiografía, mayor cantidad de neoplasias cardiacas han sido reportadas. Sin embargo, en nuestro medio son escasas las publicaciones de este tipo de tumores ${ }^{12-14}$.

Nuestros objetivos son describir las características clínicas, resultados de tratamiento quirúrgico, seguimiento y supervivencia de los pacientes operados por TCP.

\section{Método}

Se realizó un estudio analítico longitudinal de todas las cirugías realizadas por TCP en el Hospital Clínico Regional "Dr. Guillermo Grant Benavente" de Concepción-Chile tratados por nuestro equipo, en el período comprendido entre enero de 1984 y diciembre de 2019. La información se obtuvo a partir protocolos de TCP realizados al momento del ingreso al hospital, revisión de base de datos, protocolos quirúrgicos, fichas clínicas, registros de anatomía patológica y datos del Servicio de Registro Civil e Identificación de Chile.

Se ordenaron los datos mediante planilla $\mathrm{Mi}$ crosoft Excel ${ }^{\circledR}$. Se describen tipos histológicos de TCP, edad, sexo, forma de presentación clínica, localización anatómica, tratamiento quirúrgico, morbilidad, mortalidad operatoria y cirugía por recidiva en pacientes operados por TCP. Además, se comparó la supervivencia según tipo de TCP utilizando Kaplan-Meier con prueba log-rank, considerando significativo un valor $\mathrm{p}<0,05$.

Para analizar y comparar las principales características, se dividieron los TCP en 4 grupos según su frecuencia: mixomas, fibroelastomas papilares, sarcomas y misceláneos. Para la confección de la curva de supervivencia, se agruparon los TCP según histología en benignos o malignos.

El estudio y análisis de los TCP contó con la aprobación del Comité Ético Científico de nuestra institución.

\section{Resultados}

Durante todo el período se realizaron $72 \mathrm{ci}-$ rugías por TCP, correspondiendo una de ellas a una recidiva. En el período 1984-2009 (25 años) se realizaron 21 cirugías y en el período 2010-2019 (10 años) aumentó a 51 cirugías.

Las cirugías más frecuentemente realizadas fueron por tumores benignos, destacando al grupo de los mixomas con $49(68,1 \%)$ casos (Tabla 1 y Figura 1).

El grupo de los sarcomas presentó una menor edad promedio con $49 \pm 8,1$ años y se observó un predominio de las mujeres en todos los subgrupos (Tabla 2).

En cuanto a la presentación clínica, el síntoma más frecuente en todos los grupos fue la disnea y

Tabla 1. Tipos histológicos de tumores cardiacos primarios operados

\begin{tabular}{|c|c|}
\hline Tipo histológico & Total (72) \\
\hline Mixoma & $49(68,1 \%)$ \\
\hline Fibroelastoma papilar & $13(18,1 \%)$ \\
\hline $\begin{array}{l}\text { Sarcoma } \\
\text { Indiferenciado de alto grado } \\
\text { Angiosarcoma } \\
\text { Leiomiosarcoma }\end{array}$ & $\begin{array}{ll}6 & (8,3 \%) \\
3 & (4,2 \%) \\
2 & (2,8 \%) \\
1 & (1,4 \%)\end{array}$ \\
\hline $\begin{array}{l}\text { Misceláneo } \\
\text { Fibroma } \\
\text { Paraganglioma } \\
\text { Tumor del nodo AV } \\
\text { Lipoma }\end{array}$ & $\begin{array}{ll}4 & (5,6 \%) \\
1 & (1,4 \%) \\
1 & (1,4 \%) \\
1 & (1,4 \%) \\
1 & (1,4 \%)\end{array}$ \\
\hline
\end{tabular}

AV: Atrio-ventricular. 

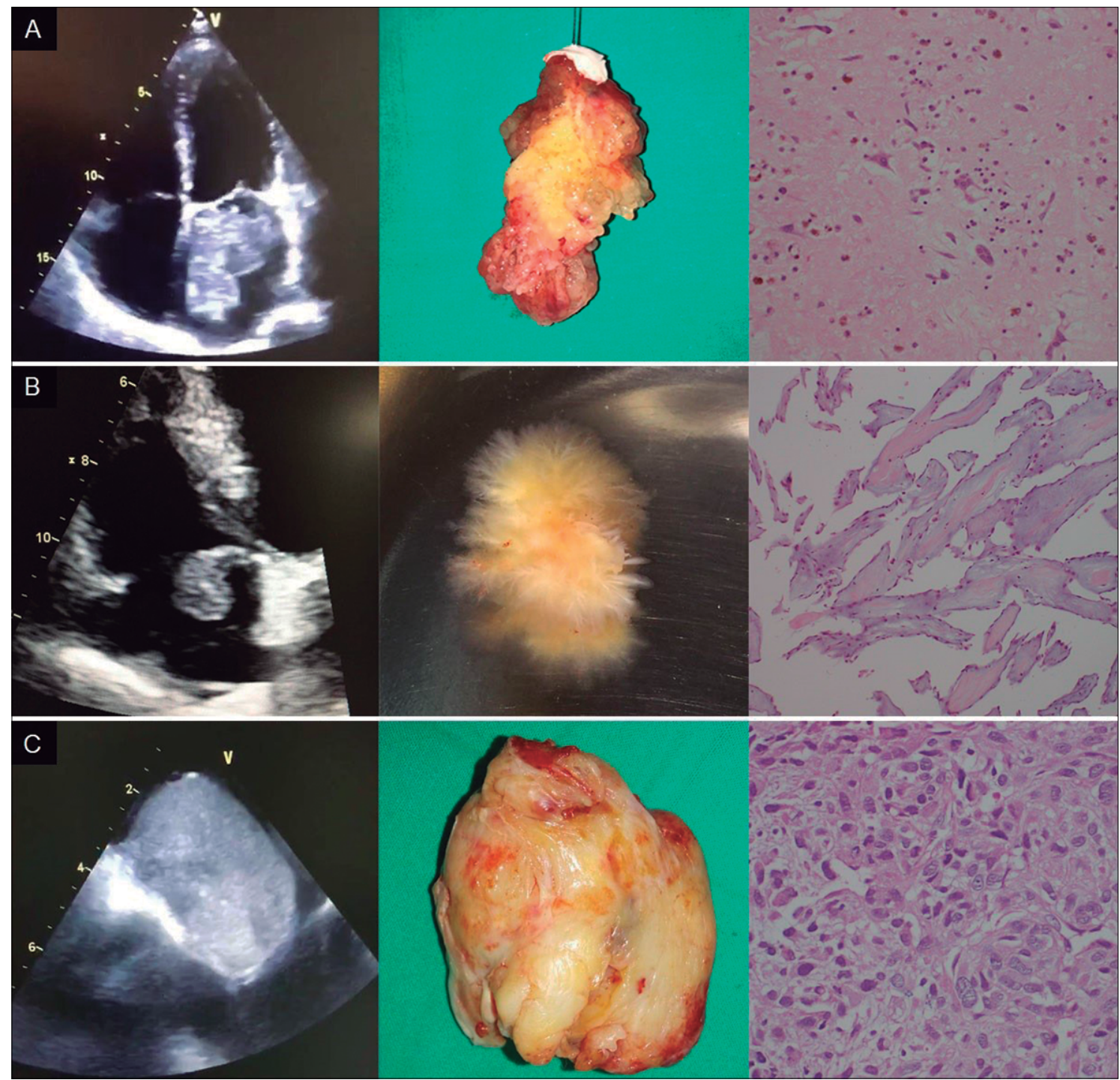

Figura 1. Tumores cardiacos primarios más frecuentes según tipo histológico: ecocardiografía, macroscopía de pieza quirúrgica e histología. A: Mixoma auricular izquierdo; B: Fibroelastoma papilar de válvula tricúspide; C: Sarcoma que ocupa aurícula izquierda.

Tabla 2. Edad y sexo en pacientes operados por tumores cardiacos primarios

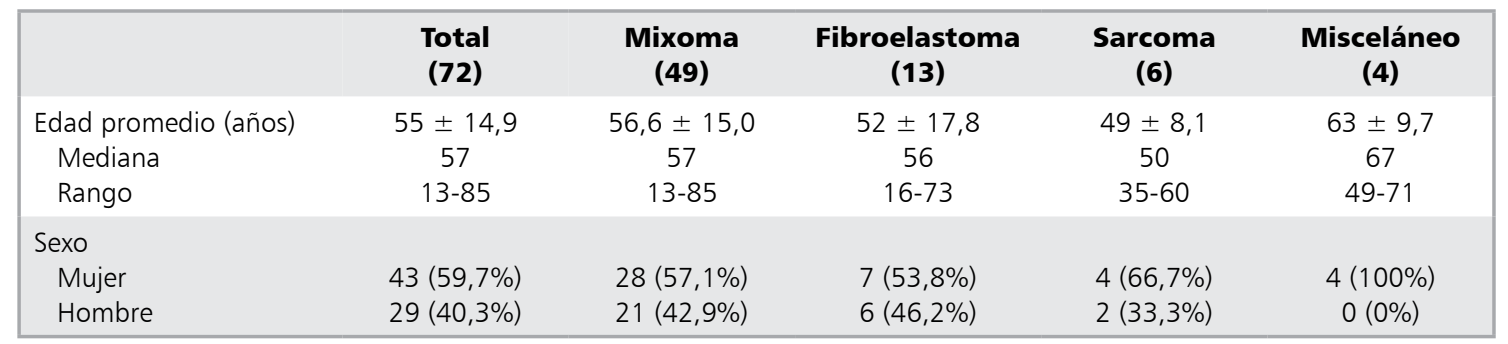


en segundo lugar destacaron los eventos neurológicos por fenómenos embólicos. En el grupo de pacientes con FP la mayoría fue asintomático $(\mathrm{Ta}-$ bla 3). El diagnóstico fue mediante ecocardiografía en todos los pacientes, existiendo $8(11,1 \%)$ casos donde se utilizó resonancia magnética, tomografía computada de tórax y/o tomografía por emisión de positrones para complementar el estudio.

Respecto a la localización anatómica de la lesión, destacó como más frecuente la aurícula izquierda con implantación en el septum interauricular en el caso de los mixomas, mientras que los FP se ubicaron preferentemente en la válvula aórtica (Tabla 4). En una mujer de 16 años el FP se localizó en la Red de Chiari, la paciente consultó por síndrome febril prolongado y soplo, la ecocardiografía informó masa móvil de 2 × 2 x 1 $\mathrm{cm}$ en aurícula derecha.

Desde el punto de vista quirúrgico, en los FP predominó la resección aislada, mientras que los mixomas y sarcomas requirieron mayormente resecciones con cierre directo del defecto o utilización de material protésico para reparación de estos defectos (parche de pericardio bovino, autólogo u otro). Un sarcoma fue irresecable y solo fue posible realizar una biopsia quirúrgica. En el 25\% de los pacientes se realizó una cirugía cardiaca asociada (Tabla 5).

Presentaron complicaciones $8(11,1 \%)$ pacientes, todos en el grupo de los mixomas. Éstas correspondieron principalmente a morbilidad de la herida operatoria (infección o dehiscencia),

Tabla 3. Presentación clínica en pacientes operados por tumores cardiacos primarios

\begin{tabular}{|c|c|c|c|c|c|}
\hline & $\begin{array}{l}\text { Total } \\
\text { (72) }\end{array}$ & $\begin{array}{l}\text { Mixoma } \\
\text { (49) }\end{array}$ & $\begin{array}{c}\text { Fibroelastoma } \\
\text { (13) }\end{array}$ & $\begin{array}{l}\text { Sarcoma } \\
\text { (6) }\end{array}$ & $\begin{array}{c}\text { Misceláneo } \\
\text { (4) }\end{array}$ \\
\hline IC (Disnea) & $31(43,1 \%)$ & $18(36,7 \%)$ & $3(23,1 \%)$ & $6(100 \%)$ & $4(100 \%)$ \\
\hline Embolia (AVE/AIT) & $13(18,1 \%)$ & $12(24,5 \%)$ & $1 \quad(7,7 \%)$ & $0 \quad(0 \%)$ & $0 \quad(0 \%)$ \\
\hline Arritmia & $4 \quad(5,6 \%)$ & $3(6,1 \%)$ & $0 \quad(0 \%)$ & $(0 \%)$ & $1 \quad(25,0 \%)$ \\
\hline Angina & $4 \quad(5,6 \%)$ & $2 \quad(4,1 \%)$ & $2(15,4 \%)$ & $(0 \%)$ & $(0 \%)$ \\
\hline Síncope & $3(4,2 \%)$ & $3 \quad(6,1 \%)$ & $0 \quad(0 \%)$ & $(0 \%)$ & $(0 \%)$ \\
\hline Fiebre & $2(2,8 \%)$ & $1 \quad(2,0 \%)$ & $1 \quad(7,7 \%)$ & $(0 \%)$ & $(0 \%)$ \\
\hline Asintomático & $23(31,9 \%)$ & $16(32,7 \%)$ & $7(53,4 \%)$ & $(0 \%)$ & $(0 \%)$ \\
\hline
\end{tabular}

IC: Insuficiencia cardiaca; AVE: Accidente vascular encefálico; AIT: Accidente isquémico transitorio.

Tabla 4. Localización anatómica de la lesión en pacientes operados por tumores cardiacos primarios

\begin{tabular}{|c|c|c|c|c|c|}
\hline & $\begin{array}{l}\text { Total } \\
(72)\end{array}$ & $\begin{array}{l}\text { Mixoma } \\
\text { (49) }\end{array}$ & $\begin{array}{c}\text { Fibroelastoma } \\
\text { (13) }\end{array}$ & $\begin{array}{l}\text { Sarcoma } \\
\text { (6) }\end{array}$ & $\begin{array}{c}\text { Misceláneo } \\
\text { (4) }\end{array}$ \\
\hline \multicolumn{6}{|l|}{ Cámaras cardiacas } \\
\hline Aurícula izquierda & $51(70,8 \%)$ & $45(91,8 \%)$ & $0 \quad(0 \%)$ & $4(66,7 \%)$ & $2(50,0 \%)$ \\
\hline Aurícula derecha & $8(11,1 \%)$ & $4 \quad(8,2 \%)$ & $0 \quad(0 \%)$ & $2(33,3 \%)$ & $2(50,0 \%)$ \\
\hline Ventrículo izquierdo & $2(2,8 \%)$ & $2 \quad(4,1 \%)$ & $0 \quad(0 \%)$ & $0 \quad(0 \%)$ & $0 \quad(0 \%)$ \\
\hline Ventrículo derecho & $2(2,8 \%)$ & $0 \quad(0 \%)$ & $0 \quad(0 \%)$ & $1(16,7 \%)$ & $1(25,0 \%)$ \\
\hline \multicolumn{6}{|l|}{ Válvulas cardiacas } \\
\hline Válvula aórtica & $10(13,9 \%)$ & $0 \quad(0 \%)$ & $10(76,9 \%)$ & $0 \quad(0 \%)$ & $0 \quad(0 \%)$ \\
\hline Válvula mitral & $1 \quad(1,4 \%)$ & $0 \quad(0 \%)$ & $1 \quad(7,7 \%)$ & $0 \quad(0 \%)$ & $0 \quad(0 \%)$ \\
\hline Válvula tricúspide & $1 \quad(1,4 \%)$ & $0 \quad(0 \%)$ & $1 \quad(7,7 \%)$ & $0 \quad(0 \%)$ & $0 \quad(0 \%)$ \\
\hline \multicolumn{6}{|l|}{ Otra } \\
\hline Red de Chiari & $1 \quad(1,4 \%)$ & $0 \quad(0 \%)$ & $1 \quad(7,7 \%)$ & $0 \quad(0 \%)$ & $0 \quad(0 \%)$ \\
\hline
\end{tabular}

*3 tumores se localizaron en más de una cámara cardiaca. 
arritmias y fenómenos embólicos. El resto de los grupos no presentó morbilidad durante su hospitalización. No existió mortalidad operatoria en la serie (Tabla 6).
La supervivencia dividida según histología entre TCP benigno y maligno se grafica en la Figura 2. Se observó diferencias estadísticamente significativas entre ambos grupos.

Tabla 5. Tipo de cirugía en pacientes operados por tumores cardiacos primarios

\begin{tabular}{|lccccc|}
\hline & $\begin{array}{c}\text { Total } \\
\mathbf{( 7 2 )}\end{array}$ & $\begin{array}{c}\text { Mixoma } \\
\mathbf{( 4 9 )}\end{array}$ & $\begin{array}{c}\text { Fibroelastoma } \\
(\mathbf{1 3 )}\end{array}$ & $\begin{array}{c}\text { Sarcoma } \\
\mathbf{( 6 )}\end{array}$ & $\begin{array}{c}\text { Misceláneo } \\
\text { (4) }\end{array}$ \\
\hline Resección aislada c/s cierre directo & $48(66,7 \%)$ & $31(63,3 \%)$ & $12(92,3 \%)$ & $2(33,3 \%)$ & $3(75,0 \%)$ \\
Resección y reparación con parche & $23(31,9 \%)$ & $18(36,7 \%)$ & $1(7,7 \%)$ & $3(50,0 \%)$ & $1(25,0 \%)$ \\
\hline Irresecable (biopsia quirúrgica) & $1(1,4 \%)$ & $0(0 \%)$ & $0(0 \%)$ & $1(16,7 \%)$ & $0(0 \%)$ \\
Cirugía cardiaca asociada & $18(25,0 \%)$ & $9(18,4 \%)$ & $6(46,2 \%)$ & $2(33,3 \%)$ & $1(25,0 \%)$ \\
$\quad$ Valvular & $11(15,3 \%)$ & $7(14,3 \%)$ & $1(7,7 \%)$ & $2(33,3 \%)$ & $1(25,0 \%)$ \\
$\quad$ Coronaria & $6(8,3 \%)$ & $2(4,1 \%)$ & $4(30,8 \%)$ & $0(0 \%)$ & $0(0 \%)$ \\
$\quad$ Cierre de CIA & $1(1,4 \%)$ & $0(0 \%)$ & $1(7,7 \%)$ & $0(0 \%)$ & $0(0 \%)$ \\
\hline
\end{tabular}

CIA: Comunicación interauricular.

Tabla 6. Morbilidad, mortalidad, estadía postoperatoria y reoperaciones por recidiva en pacientes operados por tumores cardiacos primarios

\begin{tabular}{|c|c|c|c|c|c|}
\hline & $\begin{array}{l}\text { Total } \\
(72)\end{array}$ & $\begin{array}{l}\text { Mixoma } \\
\text { (49) }\end{array}$ & $\begin{array}{l}\text { Fibroelastoma } \\
\text { (13) }\end{array}$ & $\begin{array}{l}\text { Sarcoma } \\
\text { (6) }\end{array}$ & $\begin{array}{c}\text { Misceláneo } \\
\text { (4) }\end{array}$ \\
\hline Morbilidad & $8(11,1 \%)$ & $8(16,3 \%)$ & $0(0 \%)$ & $0(0 \%)$ & $0(0 \%)$ \\
\hline Mortalidad operatoria & $0(0 \%)$ & $0(0 \%)$ & $0(0 \%)$ & $0(0 \%)$ & $0(0 \%)$ \\
\hline $\begin{array}{l}\text { Estadía postoperatoria (dí } \\
\text { Promedio } \\
\text { Mediana }\end{array}$ & $\frac{6,6 \pm 4,4}{5}$ & $\begin{array}{c}6,6 \pm 4,7 \\
5\end{array}$ & $\begin{array}{c}7,2 \pm 4,7 \\
7\end{array}$ & $5,2 \pm \frac{1,9}{5}$ & $\frac{6,8 \pm 3,9}{6}$ \\
\hline Reoperación por recidiva & $1(1,4 \%)$ & $1(2,0 \%)$ & $0(0 \%)$ & $0(0 \%)$ & $0(0 \%)$ \\
\hline
\end{tabular}

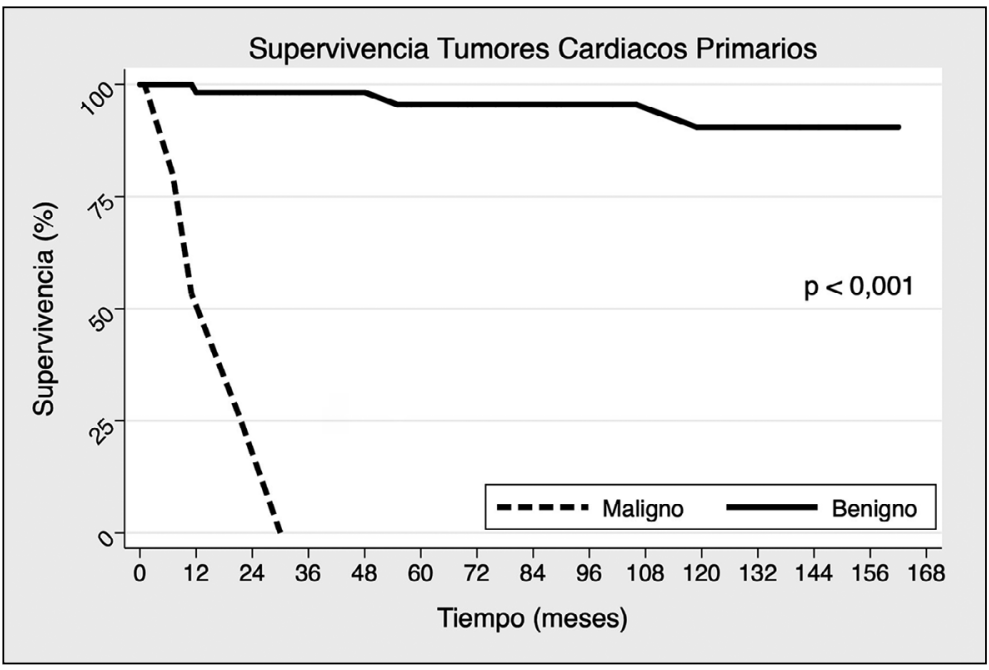

Figura 2. Supervivencia según tipo histológico de tumores cardíacos primarios. 


\section{Discusión}

En nuestra serie, los TCP son generalmente benignos y se presentan predominantemente en mujeres sobre los 50 años. El tipo histológico más frecuente es el mixoma, que se localiza principalmente en la aurícula izquierda implantado en el septum interauricular. Se diagnostican a través de ecocardiografía y su tratamiento es quirúrgico, con baja morbimortalidad operatoria. La supervivencia a largo plazo es muy diferente según tipo histológico.

Los TCP se pueden subdividir en benignos o malignos de acuerdo a la histología. Por otro lado, los tumores cardiacos secundarios, también llamados metastásicos, son por definición malignos y se originan en un sitio anatómico distinto al corazón. Diversas series reportan una frecuencia hasta 20 veces mayor de tumores secundarios por sobre los $\mathrm{TCP}^{15}$. Pese a esto, excepcionalmente son intervenidos quirúrgicamente ya que se presentan en etapas avanzadas de la neoplasia primaria por lo que poseen mal pronóstico. Las neoplasias primarias malignas que presentan más metástasis al corazón derivan del pulmón, mama, melanoma y de los linfomas ${ }^{3}$. Además, los tumores secundarios suelen ser un hallazgo en el estudio de extensión y se expresan principalmente como derrame pericárdico con o sin taponamiento cardíaco, estando la gran mayoría de ellos fuera de una opción quirúrgica, por lo que las intervenciones suelen ser de carácter paliativo ${ }^{16,17}$.

Las manifestaciones clínicas de los TCP son diversas, a menudo inespecíficas y se relacionan con el tamaño tumoral y el nivel de extensión intramural o intracavitaria que alcancen. Así un TCP de gran tamaño y de localización intracavitaria provocará manifestaciones clínicas obstructivas que deriven en sintomatología de insuficiencia cardíaca o estenosis valvular, siendo la disnea el síntoma más frecuente ${ }^{18-20}$.

Por otro lado, la infiltración de un TCP intramural al tejido eléctrico-conductor se manifestará típicamente como fenómenos arrítmicos, tales como fibrilaciones auriculares paroxísticas o bloqueos atrioventriculares completos que pueden derivar en muerte súbita ${ }^{19}$. Esto podría explicar los episodios de síncopes cardiogénicos observados en nuestra serie.

Los fenómenos embólicos son una manifestación relativamente frecuente de los TCP. La embo- lia se puede producir debido al desprendimiento de fragmentos tumorales o de trombos formados en relación al tumor, siendo el accidente cerebrovascular una complicación característica de los TCP de cavidades izquierdas ${ }^{20}$. Los fenómenos embólicos encefálicos se presentaron en $18 \%$ de los casos en nuestra serie y fueron en pacientes con mixomas o FP. Existen en la literatura reportes de otras formas de presentación, con síntomas sistémicos inespecíficos y constitucionales, tales como fiebre, baja de peso, astenia, adinamia e incluso artralgias. Estas manifestaciones sistémicas pueden ser explicadas por fenómenos inflamatorios inducidos por sustancias secretadas por el mismo tumor, incluyendo interleucinas, factor de necrosis tumoral e histamina ${ }^{21}$. En nuestra serie, solo $2(2,8 \%)$ pacientes se presentaron de esta forma, lo que creemos puede corresponder a un subdiagnóstico de los mismos, por enmascaramiento de éstos con sintomatología más dramática para el paciente, como la disnea, angina o el déficit neurológico agudo.

Respecto a la histología, el mixoma es el TCP más frecuente en el adulto y representa aproximadamente $50 \%$ de las neoplasias cardíacas benignas $^{22}$. Histológicamente corresponde a un tumor derivado de las células mesenquimales multipotenciales del subendocardio. Aunque puede afectar cualquier cámara cardiaca, la gran mayoría se localiza en la aurícula izquierda. Suelen ser móviles, pediculados con base en el tabique interauricular $y$ de tamaño variables, aunque no es infrecuente que afecten la válvula mitral con obstrucción dinámica del flujo, manifestándose con sintomatología de estenosis mitral y congestión pulmonar ${ }^{23}$. Esto se replicó en nuestra serie, ya que más de $90 \%$ de los mixomas se ubicaron en la aurícula izquierda y la mayoría de ellos presentó síntomas de insuficiencia cardiaca. La resección quirúrgica es el tratamiento de elección y los resultados muestran baja morbilidad y mortalidad, con una recidiva tumoral aproximada de $5 \%{ }^{14}$. En nuestra serie, el único paciente que se re operó correspondió a un mixoma que recidivó tras 12 años.

El FP es el segundo TCP benigno más frecuente en adultos y representa $3-10 \%$ de los $\mathrm{TCP}^{24}$. En nuestra serie los FP correspondieron a 18,6\%. Histológicamente se originan en el endocardio y su localización es característica en las válvulas cardíacas, siendo más frecuente en la válvula aórtica, seguida por la válvula mitral. Macroscópicamente se 
observa un tumor pediculado con prolongaciones papilares similares a una anémona marina ${ }^{25}$. Dado que son tumores que se ubican preferentemente en aparatos valvulares, usualmente se presentan como hallazgo incidental o como fenómeno embólico y pocas veces con clínica de insuficiencia cardiaca. $\mathrm{Al}$ ser tumores pequeños, generalmente bastan resecciones directas (shaving).

Otro de los tumores benignos presentes en nuestra serie fue el lipoma. Se caracterizan por un predominio de células adiposas benignas. Alrededor de $50 \%$ de este subgrupo de tumores se presenta en la región subendocárdica, mientras que el resto se reparte en el miocardio y subepicardio, sin embargo, también existen casos reportados en válvulas ${ }^{26,27}$. Debido a los síntomas que generan por invasión local y crecimiento progresivo, los lipomas requieren resección quirúrgica ${ }^{26-28}$.

Los paragangliomas, son tumores neuroendocrinos que pueden ser malignos o benignos $\mathrm{y}$, a su vez, hormonalmente activos o inactivos. Aquellos inactivos, es decir, los que no producen catecolaminas, provocan síntomas derivados de la compresión cardiaca o taponamiento. Mientras que aquellos con secreción hormonal generalmente pueden ocasionar síntomas sistémicos tales como cefalea, sudoración, taquicardia y palpitaciones $^{29}$. Los paragangliomas no suelen desarrollarse comúnmente en el tórax, pero cuando lo hacen, aquellos hormonalmente inactivos son más frecuentes en el pericardio, mientras que los tumores hormonalmente activos surgen con mayor frecuencia en otros sitios del tórax ${ }^{30-33}$. El caso que se presentó en nuestra serie fue el de un paraganglioma que era hormonalmente inactivo.

Aunque poco frecuentes, los fibromas son el segundo tumor cardíaco pediátrico más común e histológicamente son similares a los fibromas que surgen en otras partes del cuerpo. Generalmente proliferan en el músculo ventricular y pueden llegar a ser bastante grandes, pero a diferencia de los rabdomiomas, no regresan espontáneamente. Se originan aproximadamente cinco veces más frecuentemente en el ventrículo izquierdo que en el ventrículo derecho ${ }^{34-36}$.

El último de los tumores benignos que se presentó en nuestra serie fue el tumor quístico del nodo atrioventricular, también denominado mesotelioma del nodo atrioventricular. Corresponde a un tumor multiquístico derivado de las células del tejido de conducción y que se caracteriza por un comportamiento benigno, ser de naturaleza congénita y clásicamente se ubica en el septum interauricular. Puede causar síntomas inespecíficos, como disnea, palpitaciones u ocasionar arritmias, como el bloqueo atrioventricular completo ${ }^{37-40}$. Este tipo de tumor es muy infrecuente, nuestro caso correspondió a una mujer de 71 años, que relataba palpitaciones, la ecocardiografía describió un tumor quístico pediculado de $4 \times 3 \times 2 \mathrm{~cm}$ en aurícula derecha y que se implantaba en la base del septum interauricular.

Los TCP malignos, por otro lado, representan aproximadamente $25 \%$ de los TCP e histológicamente incluyen principalmente a los sarcomas, mesoteliomas, linfomas y teratomas malignos ${ }^{41}$. En nuestra serie solo 8,3\% de TCP fueron malignos, correspondiendo todos a sarcomas de diversos tipos.

Los angiosarcomas, corresponden al $40 \%$ de los sarcomas y se ubican generalmente en aurícula derecha, mientras que los leiomiosarcomas suelen ocupar la aurícula izquierda. Son tumores de formas y configuración variables, de rápido crecimiento y altamente infiltrativos a otras estructuras torácicas o mediastínicas, presentándose frecuentemente con derrame pericárdico hemorrágico ${ }^{42}$. En estos casos, el diagnóstico ecocardiográfico debe ir acompañado de un estudio imagenológico de extensión y etapificación que descarte invasión local y a distancia para ofrecer un tratamiento quirúrgico con intención curativa, sin embargo, muchas veces se realiza el diagnóstico en etapas tardías de la enfermedad y, por lo tanto, fuera de una opción quirúrgica.

La morbilidad operatoria es baja, siendo lo más frecuente las arritmias, en particular la fibrilación auricular, fenómenos neurológicos embólicos y complicaciones propias de la cirugía como infecciones, sangrados post quirúrgicos o morbilidad de la herida operatoria.

Aunque en nuestra serie no hubo mortalidad operatoria, se describe que ésta puede alcanzar hasta $3,29 \%{ }^{43}$, siendo más frecuente en las cirugías por TCP malignos.

La supervivencia en TCP malignos es baja (aún con tratamiento multimodal: cirugía, quimioterapia y/o radioterapia), en nuestra serie 2 pacientes fallecieron antes del año de seguimiento. La mediana de supervivencia para este tipo de TCP malignos es típicamente de 6 a 12 meses $^{44,45}$, aunque en aquellos casos que se logra resección completa 
y en sarcomas de bajo grado, pueden alcanzar una supervivencia entre 24-36 meses ${ }^{46-48}$. En los pacientes que logramos resección completa macro y microscópica (R0) la supervivencia promedio con terapia multimodal fue de 26 meses, mientras en los que la resección no fue $\mathrm{R} 0$ el promedio de supervivencia fue de 11 meses.

Por otro lado, los TCP benignos tienen una supervivencia excelente, en nuestra serie 4 pacientes fallecieron durante el seguimiento; 3 mixomas y 1 FP, la muerte fue alejada de la cirugía y por causas ajenas a la patología tumoral.

En conclusión, en nuestra serie, los TCP son predominantemente neoplasias benignas en mujeres sobre los 50 años. El más frecuente es el mixoma, que se ubica mayoritariamente en la aurícula izquierda. Se presentan frecuentemente sintomáticos, predominando la disnea y los fenómenos embólicos, aunque pueden ser asintomáticos. El método diagnóstico más importante es la ecocardiografía, sin embargo, otros estudios complementarios pueden ser requeridos. El tratamiento es quirúrgico con baja morbimortalidad. El pronóstico es excelente en los TPC benignos y pésimo en los malignos.

\section{Referencias}

1. Murphy MC, Sweeney MS, Putnam JB Jr, Walker WE, Frazier OH, Ott DA, et al. Surgical treatment of cardiac tumors: 25-year experience. Ann Thorac Surg 1990; 49: 612-8.

2. Straus R, Merliss R. Primary tumors of the heart. Arch Pathol 1945; 39: 74-8.

3. Lymburner RM. Tumors of the heart: histopathological and clinical study. Can Med Ass J 1934; 30: 368-75.

4. Lam KY, Dickens P, Chan ACL. Tumors of the heart. A 20-year experience with review of 12485 consecutive autopsies. Arch Pathol Med 1993; 117: 1027-31.

5. Hoffmeier A, Sindermann JR, Scheld HH, Martens S. Cardiac tumors-diagnosis and surgical treatment. Dtsch Arztebl Int 2014; 111: 205-11.

6. Petris AO, Alexandrescu DM, Costache II. Cardiac tumors. Rev Med Chir Soc Med Nat Iasi 2014; 118: 28992.

7. Nehaj F, Sokol J, Mokan M, Jankovicova V, Kovar F, Kubaskova M, et al. Outcomes of Patients with Newly Diagnosed Cardiac Myxoma: A Retrospective Multicentric Study. Biomed Res Int 2018; Article ID 8320793: e1-e5.
8. Wang JG, Wang B, Hu Y, Liu JH, Liu B, Liu H, et al. Clinicopathologic features and outcomes of primary cardiac tumors: a 16-year-experience with 212 patients at a Chinese medical center. Cardiovasc Pathol 2018; 33 : 45-54.

9. Abad C. Tumores cardíacos (I). Generalidades. Tumores primitivos benignos. Rev Esp Cardiol 1998; 51: 10-20.

10. Meng Q, Lai H, Tong W, Qian Y, Lai S. Echocardiographic and pathologic characteristic of primary cardiac tumors: a study of 149 cases. Int J Cardiol 2002; 84: 69-75.

11. Saad AM, Abushouk AI, Al-Husseini MJ, Salahia S, Alrefai A, Afifi AM, et al. Characteristics, survival and incidence rates and trends of primary cardiac malignancies in the United States. Cardiovasc Pathol 2018; 33: 27-31.

12. González R, Raffo M, Alarcón E, Gyhra A, Saldías R, Stockins A, et al. Tumores cardíacos. Rev Chil Cir 2005; 57: 195-8.

13. Arnaiz P, Toledo I, Borzutzky A, Urcelay G, Heusser F, Garay F, et al. Comportamiento clínico de los tumores cardíacos desde el feto hasta el adulto: serie multicéntrica de 38 pacientes. Rev Med Chile 2006; 134: 113545.

14. Becker P, Ramírez A, Zalaquett R, Moran S, Irarrázabal $\mathrm{M}$, Arretz C, et al. Mixoma cardiaco: caracterización clínica, métodos diagnósticos y resultados alejados del tratamiento quirúrgico durante tres décadas de experiencia. Rev Med Chile 2008; 136: 287-95.

15. Reynan K. Frequency of primary tumors of the heart. Am J Cardiol 1996; 77: 107.

16. Burnside N, Macgowan SW. Malignant primary cardiac tumors. Interact Cardiovasc Thorac Surg 2012; 15: 1483-9.

17. Yu L, Gu T, Shi E, Ziu Z, Fang Q, Wang C, et al. Primary malignant cardiac tumors. J Cancer Res Clin Oncol. 2014; 140: 1047-55.

18. González R, Farías J, Seguel E, Stockins A, Álvarez R, Neira L, et al. Mixoma cardíaco y enfermedad coronaria en paciente octogenario. Rev Chil Cir 2009; 61: 221-2.

19. Roberts WC. Primary and secondary neoplasms of the heart. Am J Cardiol 1997; 80: 671-82.

20. Córdova S, Barrero R. Tumores cardíacos. En: Bravo C, Editora. Enfermedades del corazón y de los grandes vasos. 4ta edición. Santiago de Chile. Editorial Mediterráneo; 2013. p. 1193-201.

21. Saji T, Yanagawa E, Matsuura H, Yamamoto S, Ishikita T, Matsuo N, et al. Increased serum interleukin-6 mRNA in cardiac myxomas. Am Heart J 1991; 122: 57980.

22. Masuda I, Ferreño A, Pasca J, Pereiro G, Lastiri H. Tu- 
mores cardíacos primarios. Mixoma auricular. Rev Fed Arg Cardiol 2004; 33: 196-204.

23. Gabe E, Rodríguez C, Vigliano C, San Martino J, Wisner J, González P, et al. Mixomas cardíacos: correlación anatomoclínica. Rev Esp Cardiol 2002; 55: 505-13.

24. Gowda R, Khan I, Nair C, Mehta N, Vasavada B, Sacchi T. Cardiac papillary fibroelastoma: A comprehensive analysis of 725 cases. Am Herat J 2003; 146: 404-10.

25. González R, Reyes R, Riquelme A, Seguel E, Stockins A, Jadue A, et al. Accidente cerebrovascular embólico secundario a fibroelastoma papilar de válvula mitral. Rev Med Chile 2019; 147: 243-6.

26. Benvenuti LA, Mansur AJ, Lopes DO, Campos RV. Primary lipomatous tumors of the cardiac valves. South Med J 1996; 89: 1018-20.

27. Hananouchi GI, Goff WB 2nd. Cardiac lipoma: six-year follow-up with MRI characteristics, and a review of the literature. Magn Reson Imaging 1990; 8: 825-8.

28. Rehman A, Heng EE, Cheema FH. Calcified amorphous tumour of right ventricle. Lancet 2014; 383: 815.

29. Dumantepe M, Ak K, Mungan U, Alp I, Inan BK, Yilmaz AT. Blood cyst of the right ventricle presenting as recurrent high fever and chills in an adult. Ann Thorac Surg 2009; 87: 638-40.

30. Biology and treatment of thoracic tumors of neural crest origin. In: Thoracic Oncology, WB Saunders, Philadelphia 1989. p.520.

31. Tanaka F, Kitano M, Tatsumi A, Huang C, Nagasawa M, Mino M, et al. Paraganglioma of the posterior mediastinum: value of magnetic resonance imaging. Ann Thorac Surg 1992; 53: 517-9.

32. Flickinger FW, Yuh WT, Behrendt DM. Magnetic resonance imaging of mediastinal paraganglioma. Chest 1988; 94: 652-4.

33. ElBardissi AW, Dearani JA, Daly RC, Mullany CJ, Orszulak TA, Puga FJ, et al. Analysis of benign ventricular tumors: long-term outcome after resection. J Thorac Cardiovasc Surg 2008; 135: 1061-8.

34. Valente M, Cocco P, Thiene G, Casula R, Poletti A, Milanesi $\mathrm{O}$, et al. Cardiac fibroma and heart transplantation. J Thorac Cardiovasc Surg 1993; 106: 1208-12.

35. Bapat VN, Varma GG, Hardikar AA, Sivaraman A, Agrawal NB, Tendolkar AG. Right-ventricular fibroma presenting as tricuspid stenosis- case report. Thorac Cardiovasc Surg 1996; 44: 152-4.

36. Burke AP, Rosado de Christenson M, Templeton PA,
Virmani R. Cardiac fibroma: clinicopathologic correlates and surgical treatment. J Thorac Cardiovasc Surg 1994; 108: 862-70.

37. Ford SE. Congenital cystic tumors of the atrioventricular node successful demonstration by an abbreviated dissection of the conduction system. Cardiovasc Pathol 1999; 27: 161-7.

38. Balasundaram S, Halees SA, Duran C. Mesothelioma of the atrio-ventricular node first successful follow-up after excision. Eur Heart J 1992; 13: 718-9.

39. Saito S, Kobayash J, Tagusari O, Bando K, Niwaya K, Nakajima $H$, et al. Successful excision of a cystic tumor of the atrioventricular nodal region. Circ J 2005; 69: 1293-4.

40. Kelsey BL, Tianshu F, Vidhya N, Cusimano RJ, Butanu J. Cystic tumor of the atrioventricular node: A rare antemortem diagnosis. Cardiovasc Pathol 2012; 21: 120-7.

41. Abad C. Tumores cardíacos (II). Tumores primitivos malignos. Tumores metastásicos. Tumor carcinoide. Rev Esp Cardiol 1998; 51: 103-14.

42. Saraiva J, Antunes PE, Carvalho L, Antunes MJ. Primary malignant cardiac tumors: Surgical results. Rev Port Cardiol 2016; 35: 199-204.

43. Baikoussis NG, Papakonstantinou NA, Dedeilias P, Argiriou M, Apostolakis E, Koletsis E, et al. Cardiac tumors: a retrospective multicenter institutional study. J BUON 2015; 20: 1115-23.

44. Burke AP, Cowan D, Virmani R. Primary sarcomas of the heart. Cancer 1992; 69: 387-95.

45. Truong PT, Jones SO, Martens B, Alexander C, Paquette $\mathrm{M}$, Joe $\mathrm{H}$, et al. Treatment and outcomes in adult patients with primary cardiac sarcoma: the British Columbia Cancer Agency experience. Ann Surg Oncol 2009; 16: 3358-65.

46. Zhang PJ, Brooks JS, Goldblum JR, Yoder B, Seethala R, Pawel B, et al. Primary cardiac sarcomas: a clinicopathologic analysis of a series with follow-up information in 17 patients and emphasis on long-term survival. Hum Pathol 2008; 39: 1385-95.

47. Putnam Jr JB, Sweeney MS, Colon R, Lanza LA, Frazier $\mathrm{OH}$, Cooley DA. Primary cardiac sarcomas. Ann Thorac Surg 1991; 51: 906-10.

48. Shapira OM, Korach A, Izhar U, Koler T, Wals O, Ayman $\mathrm{M}$, et al. Radical multidisciplinary approach to primary cardiac sarcomas. Eur J Cardiothorac Surg 2013; 44: 330-6. 